\title{
Uma sequência didática e suas representações: estudo das operações com arcos no ensino médio

\author{
A didactic sequence and its representations: study of archery \\ operations in high school
}

\author{
SILVIA TERESINHA FRIZZARINI ${ }^{1}$ \\ CLAUDETE CARGNIN ${ }^{2}$
}

\begin{abstract}
Resumo
Esse trabalho trata do ensino de operações com arcos no Ensino Médio e suas Representações Semióticas. O embasamento teórico utilizado foi a teoria de Duval, a partir uma sequência de didática no Geogebra, em que foram analisadas a abordagem da operação de arcos comumente adotada no Ensino Médio e suas questões históricas. Um roteiro da sequência didática, em um ambiente de Geometria Dinâmica, é sugerido com a finalidade de trabalhar com mais detalhes as concepções sobre Trigonometria e a ênfase em suas conversões de registros, segundo a teoria adotada. A análise qualitativa da sequência didática sugere que a apresentação, com os tipos de representação pouco utilizados de forma geral na escola hoje, favorece diversos fatores relativos ao conceito de soma de arcos. Concluímos que uma representação que evidencie a soma de arcos, assim como a conversão de registros, pode favorecer um melhor entendimento dos objetos com os quais se está operando e explicitando propriedades inerentes aos mesmos.
\end{abstract}

Palavras-chave: Soma de arcos. Representação semiótica. Ensino Médio. Geogebra

\section{Resumen}

Este trabajo trata de la enseñanza de operaciones con arcos en la Enseñanza Media y sus Representaciones Semióticas. La base teórica utilizada fue la teoría de Duval, a partir de una secuencia de didáctica en GeoGebra, en la que se analizaron el enfoque de la operación de arcos comúnmente adoptada en la Enseñanza Media y sus cuestiones históricas. Un guión de la secuencia didáctica, en un ambiente de Geometría Dinámica, es sugerido con la finalidad de trabajar con más detalles las concepciones sobre Trigonometría y el énfasis en sus conversiones de registros, según la teoría adoptada. El análisis cualitativo de la secuencia didáctica sugiere que la presentación, con los tipos de representación poco utilizados de forma general en la escuela hoy, favorece diversos factores relativos al concepto de suma de arcos. Concluimos que una representación que evidencie la suma de arcos, así como la conversión de registros, puede favorecer un

\footnotetext{
${ }^{1}$ Universidade do Estado de Santa Catarina - UDESC, Joinville -Brasil, stfrizzarini@ hotmail.com

${ }^{2}$ Universidade Tecnológica Federal do Paraná - UTFPR, Campo Mourão - Brasil, cargnin@utfpr.edu.br
} 
mejor entendimiento de los objetos con los que se está operando y explicitando propiedades inherentes a los mismos.

Palabras clave: Suma de arcos. Representación semiótica. Enseñanza Media. Geogebra

\title{
Résumé
}

Ce travail porte sur l'enseignement des opérations avec arcs au lycée et ses représentations sémiotiques. La base théorique utilisée était la théorie de Duval, à partir d'une séquence didactique dans GeoGebra, dans laquelle l'approche de l'opération des arcs couramment adoptée au lycée et ses questions historiques ont été analysées. Un script de la séquence didactique dans un environnement de géométrie dynamique est proposé dans le but de travailler plus en détail sur les concepts de la trigonométrie et l'accent mis sur ses conversions de registres selon la théorie adoptée. L'analyse qualitative de la séquence didactique suggère que la présentation, avec les types de représentation peu utilisés en général dans l'école aujourd'hui, favorise plusieurs facteurs liés au concept de somme des arcs. Nous concluons qu'une représentation qui met en évidence la somme des arcs, ainsi que la conversion des registres, peut favoriser une meilleure compréhension des objets avec lesquels on opère et expliquer les propriétés inhérentes à ceux-ci.

Mots-clés : Somme des arcs. Représentation sémiotique Lycée Geogebra

\begin{abstract}
This work deals with the teaching of operations with arcs in High School and its Semiotic Representations. The theoretical basis used was the Duval theory, starting from a didactic sequence in GeoGebra, in which the approach of the arcs operation commonly adopted in High School and its historical questions were analyzed. A script of the didactic sequence in an environment of Dynamic Geometry is suggested for the purpose of working in more detail on the concepts of Trigonometry and the emphasis on its conversions of registers according to the theory adopted. The qualitative analysis of the didactic sequence suggests that the presentation, with the types of representation little used in general in the school today, favors several factors related to the concept of sum of arcs. We conclude that a representation that evidences the sum of arcs, as well as the conversion of registers, can favor a better understanding of the objects with which one is operating and explaining properties inherent thereto.
\end{abstract}

Keywords: Sum of arcs. Semiotic representation. High school. Geogebra

\section{Introdução}

Segundo a Teoria de Registro de Representação Semiótica (TRRS), os objetos abstratos com que a matemática trabalha são acessíveis apenas por meio de suas representações semióticas. Entretanto, nem todos os sistemas semióticos são capazes de produzir a 
apreensão conceitual necessária à aprendizagem matemática, o que requer algumas especificidades que apenas os Registros de representação semiótica têm.

Os registros são sistemas semióticos criadores de novos conhecimentos. Para ser um registro, um sistema semiótico deve cumprir duas condições. Primeiramente, poder produzir representações que permitem tanto ter acesso a objetos perceptivamente ou instrumentalmente inacessíveis, quanto explorar tudo o que é possível. Em seguida, e sobretudo, abrir um campo de operações específicas que permitem transformar as representações produzidas em novas representações (DUVAL, 2011, p. 97).

Os registros considerados por Duval (2011) são as línguas, figuras, gráficos etc.

O estudo de arcos requer transitar em vários registros, isso porque a geometria é muito utilizada no estudo da trigonometria, “[...] a trigonometria nasceu como uma ferramenta da geometria para auxiliar nos problemas de navegação e astronomia (JANOS, 2011, p. 127)". Segundo Janos (2011), enquanto que na geometria podemos desenhar um triângulo a partir de dois ângulos e um lado, na trigonometria o interesse é em determinar esses lados e ângulos do triângulo de maneira algébrica.

Conhecer o conceito de ângulos, a partir de seus lados, é fundamental no estudo das operações com arcos; conteúdo que faz parte da trigonometria trabalhada no Ensino Médio e que faz constantes associações à geometria trabalhada no Ensino Fundamental. A história sobre ângulos remonta ao menos 190-120 a.C., com o astrônomo grego Hiparco de Rode, entre outros matemáticos gregos. Segundo Berlingoff e Gouvêa (2008), para determinar as posições de astros e planetas, os gregos relacionavam um ângulo central $\beta$ em um círculo, de raio fixo, com um segmento (de reta) específico, ou seja, sua corda, conforme a Figura 1.

Figura 1: A corda de um ângulo

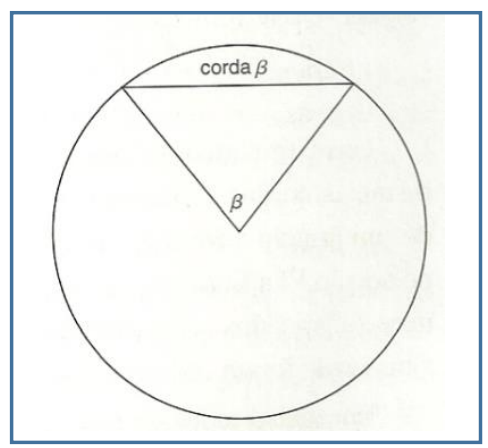

Fonte: Berlingoff e Gouvêa (2008, p. 189)

Podemos observar que, desde os tempos antigos, os diferentes registros eram fundamentais para esse conteúdo, em que os gregos sempre se apoiavam no registro 
geométrico para descrever os fenômenos físicos da natureza. De acordo com a Teoria das Representações Semióticas (DUVAL, 2009), um mesmo objeto tem várias representações, em diferentes registros, sendo que cada representação fornece uma informação parcial acerca do objeto. Por exemplo, ao considerarmos o objeto "arco" podemos escrever as seguintes representações em diferentes registros: (1) linguística (registro em língua natural) - é um segmento de uma curva ${ }^{3}$; (2) algébrica (registro algébrico) - pode ser designada por sen, $\cos$ ou $\operatorname{tg}$ de um ângulo $\beta$ qualquer, ou ainda, (3) gráfica (registro gráfico) - relação do ângulo central $\beta$ em um círculo (Figura 1). Permanecer num único registro de representação significa tomar a representação como sendo, de fato, o objeto matemático - o que pode incorrer em erros conceituais; no caso do exemplo acima, sen $\beta$ seria o próprio objeto "arco" e não a sua representação algébrica matemática. Segundo Duval (2009), para não confundir o objeto e o conteúdo de sua representação, é necessário dispor de, ao menos, duas representações, de modo que ambas as representações devam ser percebidas como representando o mesmo objeto. Além disso, é preciso que o estudante seja capaz de transitar espontaneamente entre uma e outra representação.

Diante do exposto, a seguinte questão é posta: Como potencializar o estudo das operações com arcos para que o aluno transite em diferentes representações? $\mathrm{Na}$ tentativa de responder esta pergunta, este trabalho tem por objetivo apresentar e discutir uma sequência didática para o ensino de operações com arcos e suas Representações Semióticas no Ensino Médio.

\section{Operações com arcos}

O estudo das operações com arcos auxilia o cálculo de funções circulares cujos arcos não sejam os conhecidos $30^{\circ}, 45^{\circ}$ e $60^{\circ}$. Imagine que se tenha que calcular o valor do $\cos 90^{\circ}$, mas não tem em mente o ciclo trigonométrico, nem uma calculadora científica, apenas uma tabela básica com a representação numérica de valores para seno e cosseno dos ângulos $30^{\circ}, 45^{\circ}$ e $60^{\circ}$. Como calcular então?

Sabemos que: $\quad \cos 30^{\circ}=\frac{\sqrt{3}}{2}$ e $\cos 60^{\circ}=\frac{1}{2}$

\footnotetext{
${ }^{3}$ Segundo o minidicionário Aurélio (2000)
} 
O aluno pode pensar, de maneira equivocada, na soma dos ângulos $60^{\circ}+30^{\circ}=90^{\circ}$ para calcular o valor do $\cos 90^{\circ}$. No entanto, a soma do cosseno de cada um desses ângulos não é equivalente ao cosseno da soma desses ângulos, note:

$$
\cos 60^{\circ}+\cos 30^{\circ} \neq \cos 90^{\circ}
$$

Podemos perceber que se $\mathrm{a}=60^{\circ}$ e $\mathrm{b}=30^{\circ}$, então:

$$
\cos a+\cos b \neq \cos (a+b)
$$

Outro fator importante para se destacar é quando precisamos calcular a diferença de dois desses ângulos que já conhecemos. Observe a Figura 2, onde o ponto P representa a única posição de uma pessoa, em um dos lados do terreno arenoso do rio, de onde é possível chegar à uma ilha situada no meio do rio. O ponto $\mathrm{F}$ representa um farol de navegação, situado na ilha, cuja distância até a pessoa é de $1 \mathrm{~km}$.

\section{Figura 2: situação hipotética}

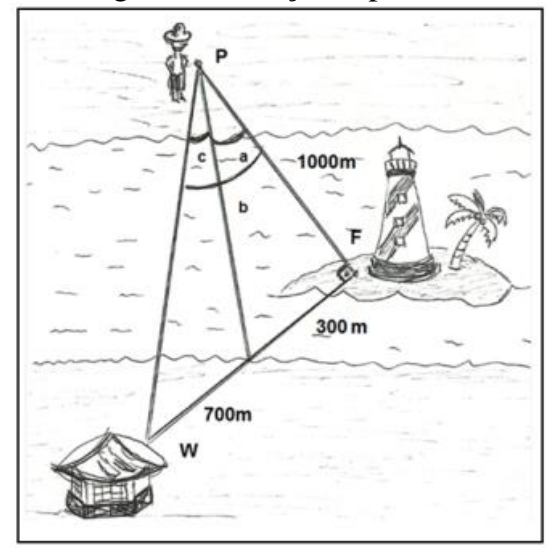

Fonte: Frizzarini (2018, p. 188)

Para chegar a uma casa situada do outro lado do rio, em linha reta a partir do farol, é preciso percorrer uma distância de 300m até a beira do rio mais uma distância de $700 \mathrm{~m}$ até a casa, num ângulo de $90^{\circ}$ entre a direção da pessoa ao farol e do farol à casa, conforme apresentado na Figura 2. A pessoa, que está de um lado do rio, precisa encontrar cada um dos arcos a, b e c para chegar até casa, do outro lado do rio, sem ter que passar pela ilha.

Além da representação de uma situação real, temos aí a representação de dois triângulos retângulos em F com as medidas dos seus catetos, podendo, assim, obter os valores dos $\operatorname{arcos}$ "a" e "b", de maneira algébrica, com o cálculo das tangentes que nada mais é que a razão trigonométrica entre o cateto oposto e o cateto adjacente, portanto:

$$
\operatorname{tg} \mathrm{a}=300 / 1000=0,3
$$




$$
\operatorname{tg} b=1000 / 1000=1
$$

No entanto, não poderíamos encontrar o valor do arco c do mesmo modo, pois esse arco não faz parte de um triângulo retângulo. Muito menos subtrair o valor dos arcos a de $b$, ou seja, $\operatorname{tg} b-\operatorname{tg} a \neq \operatorname{tg}(b-a)$, como exposto antes com $\cos a+\cos b \neq \cos (a$ $+b)$.

Para resolver esses casos, montou-se uma sequência didática para estudar, de maneira dinâmica no GeoGebra e com distintas representações, as funções trigonométricas de arcos cujas medidas podem ser colocadas na forma de soma $(a+b)$ ou subtração $(\mathrm{a}-\mathrm{b})$ de dois números quaisquer $\mathrm{a} e \mathrm{~b}$, ou seja: sen $(\mathrm{a} \pm \mathrm{b}) \cos (\mathrm{a} \pm \mathrm{b}), \operatorname{tg}(\mathrm{a} \pm$ b), entre outras formas.

\section{Apresentação e discussão da sequência didática}

A sequência didática aqui apresentada foi aplicada em uma turma de segundo ano de um curso técnico integrado em informática, no ano de 2015. A construção requerida (vide quadros 1 de 2) foi realizada extraclasse. Em sala de aula, destinou-se o tempo para discussão das dúvidas e do conteúdo em si (soma de arcos). O referencial teórico adotado nesta investigação foi a "Teoria dos Registros de Representações Semióticas", de Duval (2009; 2011). Na maioria de seus trabalhos, o autor chama a atenção para a importância em distinguir um objeto de sua representação. Os objetos matemáticos, que não são acessíveis pela percepção, têm representações diferentes, dependendo da necessidade e do uso.

Acredita-se que a conversão depende de uma compreensão conceitual, isto é, de uma atividade "puramente mental". Essa atividade mental, além de abstrair o objeto matemático, quando da relação entre representação e referência, permitirá apreendê-lo, independentemente da representação que se use.

Duval (2009) caracteriza as representações segundo as oposições: "interna/externa" e “consciente/não-consciente" (p. 40), conforme a tabela 1 abaixo:

Tabela 1: Tipos de representações

\begin{tabular}{|l|l|l|}
\hline & INTERNA & EXTERNA \\
\hline CONSCIENTE & Mental & Semiótica \\
\hline NÃO-CONSCIENTE & Computacional & \\
\hline
\end{tabular}

Fonte: DUVAL, 2009, p. 43 
Dentre essas oposições, é possível distinguir três tipos de representações que podem ser mobilizadas por quaisquer atividades matemáticas: mental, semiótica e computacional, umas com mais eficiência que as outras, que fizeram parte de nossa sequência didática. A tarefa, apresentada nos Quadros 1 e 2, serviu como elemento motivador da aula sobre o tema "arcos", cuja construção resulta em algo do tipo que é apresentado na Figura 3.

Figura 3: Representação geométrica da soma de arcos na Tarefa do Quadro 1

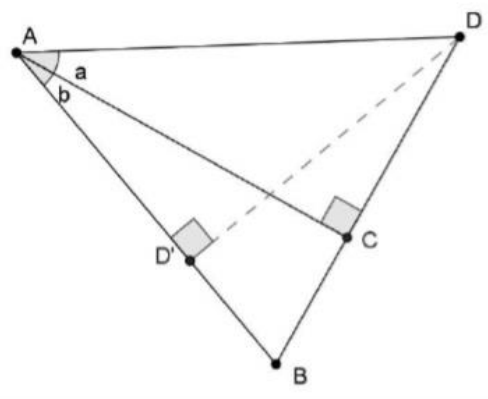

Fonte: Frizzarini (2018, p.190).

Quadro 1: Construção do seno da soma de dois ângulos no GeoGebra - parte 1

1. Abra um arquivo no Geogebra e crie uma reta $r$ e uma perpendicular (a $r$ ) $s$. Chame a intersecção dessas retas de $\mathrm{C}$.

2. Marque um ponto $\mathrm{A}$ em $r$, diferente do ponto $\mathrm{C}$.

3. Marque dois pontos $\mathrm{B}$ e $\mathrm{D}$ na reta $s$, situados em lados distintos em relação à reta $r$.

4. Esconda as retas $r$ e $s$ (basta clicar sobre o botão correspondente na janela de álgebra) e desenhe os segmentos $\mathrm{AB}, \mathrm{AC}, \mathrm{AD}$ e $\mathrm{BD}$.

5. Calcule a medida do ângulo CÂD, com nome "a", faça o mesmo no ângulo BÂC com nome "b" e coloque cores diferentes para cada ângulo. Use o ícone $\stackrel{\alpha}{*}$. Lembrese de clicar sobre os pontos na ordem em que eles aparecem no texto, ou seja, para medir $C \hat{A} D$, clique primeiro em $C$, depois em $A$, e por último em $D$.

6. Crie uma perpendicular ao lado $\mathrm{AB}$, passando pelo ponto $\mathrm{D}$, marcando o ponto de interseção D'. Esconda a reta perpendicular e crie o segmento DD' na cor cinza e estilo tracejado, de tal forma que D' se localize entre A e B. Movimente se necessário (Figura $3)$.

Fonte: Frizzarini (2018, p. 189) 
Em sala de aula, essa construção permitiu que os estudantes "acompanhassem", sob duas perspectivas - algébrica/numérica e gráfica, os elementos abstratos envolvidos, graças às funcionalidades do Geogebra, que mostra, na janela de álgebra, as representações algébricas/numéricas dos objetos, ao mesmo tempo em que proporciona uma "visualização" desses elementos, corroborando o que afirma Duval: As representações semióticas "permitem uma visão do objeto através da percepção de estímulos (pontos, traços, caracteres,...), tendo valor de significante" (DUVAL, 2009, p. 44). O fato de os alunos poderem confrontar as representações de forma instantânea permitiu que questionamentos e inferências mais coerentes e consistentes fossem realizados.

Quadro 2: Construção do seno da soma de dois ângulos no GeoGebra - parte 2

7. Para o triângulo ADD', realize os cálculos para sen $(\mathrm{a}+\mathrm{b})=\frac{\overline{D D^{\prime}}}{\overline{A D}}$, digitando na caixa de entrada “distância DD'/distância AD”. Crie um campo de texto clicando $A B C$ na barra de ferramenta e em seguida num lugar vazio da janela gráfica. Para que o texto seja dinâmico, insira a parte estática do texto usando o teclado (ex.: sen $(a+b)=)$ e em seguida clique no objeto (que aparece na janela de álgebra) cujo valor pretende mostrar no texto, ou seja, o cálculo distância DD'/distância AD. O GeoGebra adiciona automaticamente a sintaxe ("sen $(a+b)="$ distância DD'/distância AD ).

8. Para os triângulos ACD e ACB, calcule os valores de sen(a), $\cos (a) \operatorname{sen}(b)$ e cos(b), utilizando respectivamente as razões trigonométricas dos seus correspondentes segmentos e expresse em campos de textos dinâmicos com legendas, conforme explicado no passo 7. $(\operatorname{sen}(a)=\operatorname{Distância}[D, C] / D i s t a ̂ n c i a[A, D] ; \cos (a)=\operatorname{Distância}[A$, C] / Distância[A, D]; sen(b)= Distância[B, C] / Distância[A, B]; cosb= Distância[A, C]/Distância[A, B])

9. Calcule e expresse o valor das expressões " $\operatorname{sen}(a) * \cos (b)+\operatorname{sen}(b) * \cos (a)="$ e "sen(a)+sen(b)" num campo de texto, ao lado de "sen $(a+b)="$ usando as respectivas razões trigonométricas criadas no passo 8 (quando for escrever a fórmula, utilize a letra que aparece na janela de álgebra para designar cada uma das razões).

Fonte: Frizzarini (2018, p. 191).

Seguindo os passos descritos no Quadro 2, a construção obtida é algo como apresentado na Figura 4.

Figura 4: Janela de visualização do Geogebra. 


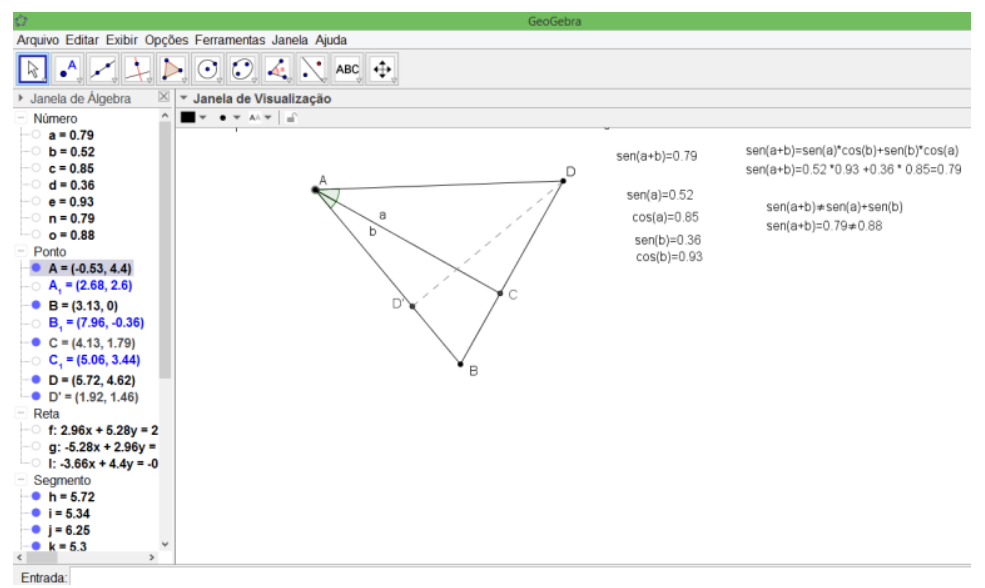

Fonte: Frizzarini (2018, p. 191).

Observe que a Figura 4 favorece o tratamento tanto no registro algébrico (que pode ser observado no texto escrito ao lado da figura), quanto no registro gráfico, ao mesmo tempo em que permite "visualizar" a conversão entre as representações. Em sala de aula, muitos estudantes iam arrastando os pontos do triângulo original para "testar" a validade da identidade. Ao fazer isto, os estudantes estavam se defrontando com as atividades cognitivas de tratamento e conversão, definidas por Duval (2009) como:

[...] Tratamento é a transformação de uma representação obtida como dado inicial em uma representação considerada como terminal em relação a uma questão, a um problema ou a uma necessidade, os quais fornecem o critério de parada na série de transformações efetuadas (DUVAL, 2009, p. 57).

[...] Converter é transformar a representação de um objeto, de uma situação ou de uma informação dada num registro em uma representação desse mesmo objeto, dessa mesma situação ou da mesma informação num outro registro (DUVAL, 2009, p. 58).

Neste processo, a representação mental é mobilizada a partir de "todo o conjunto de imagens e de conceituações que um indivíduo pode ter sobre o objeto, sobre sua situação e sobre aquilo que lhe é associado" (DUVAL, 2009, p. 15).

A partir dessa construção, foi possível desafiar os estudantes a demonstrarem a proposição: $\operatorname{sen}(a+b)=\operatorname{sen}(a) \cdot \cos (b)+\operatorname{sen}(b) \cdot \cos (a)$. Uma sugestão dada a eles foi para utilizar as razões trigonométricas da construção realizada no Geogebra de $\operatorname{sen}(a+b)$ e de $\operatorname{sen}(a) \cos (b)+\operatorname{sen}(b) \cos (a)$, para realizar o cálculo da área do triângulo $\mathrm{ABD}$ de duas maneiras distintas e fazer as devidas substituições (a demonstração não será realizada aqui, mas pode ser encontrada em Frizzarini (2018)). Observe que, desta forma, os alunos acabaram assumindo uma responsabilidade que, em geral, é do professor: demonstrar as propriedades matemáticas envolvidas no contexto em estudo. 
Neste momento, as representações computacionais mobilizadas "são todas aquelas cujos significantes, de natureza homogênea, não requerem visão de objeto, e que permitem uma transformação algorítmica de uma sucessão de significantes em uma outra” (DUVAL, 2009, p. 47). Para Duval (2009), as mudanças de representação, quando existe a coordenação destas representações, são realizadas de forma automática, como se estivessem num nível de não-consciência, ou seja, elas correspondem à familiaridade ou à experiência de uma prática ou de uma competência adquirida, o que "[...] corresponde a um processo de objetivação" (DUVAL, 2009, p. 41). Assim, a descoberta daquilo que o sujeito não supunha, mesmo com explicações, só é possível com o que Duval (2009) chama por função de objetivação, possíveis por meio das representações internas (mental) ou das representações externas (semiótica).

$\mathrm{Na}$ tarefa aqui apresentada, foi possível mobilizar diferentes registros de representação semiótica, de forma simultânea, propiciada pela utilização do software Geogebra, sendo: algébrico (nas fórmulas que envolve o tema), numérico (nas operações envolvendo as medidas dos segmentos) e gráfica (na imagem construída). Essa mobilização permitiu que os estudantes não vissem a fórmula do seno da soma de dois arcos, por exemplo, apenas como uma fórmula, mas como uma relação entre medidas na qual os ângulos não podem ser omitidos quando representados algebricamente.

\section{Considerações finais}

Apesar das representações internas (mental) ou das representações externas (semiótica) preencherem a função de objetivação, as representações (externas) semióticas, por serem diretamente visíveis e observáveis, preenchem ainda as funções de expressão e de tratamento intencional, descritas e contextualizadas na sequência didática apresentada. A variedade de registros, segundo Duval (2009), raramente é levada em conta no ensino e, para poder estar em posição de observá-la, é preciso começar por distinguir bem os dois tipos de transformações: interna ao registro (tratamento) e externa ao registro (conversão), e suas contribuições à aprendizagem matemática.

$\mathrm{Na}$ tarefa aqui apresentada, a utilização do Geogebra possibilitou a mobilização das representações algébrica e gráfica e favoreceu o tratamento algébrico para a demonstração da fórmula: $\operatorname{sen}(a+b)=\operatorname{sen}(a) \cdot \cos (b)+\operatorname{sen}(b) \cdot \cos (a)$ por parte dos alunos, além de questionamentos estudantis em nível mais elevado que o observado pelas autoras em salas nas quais essa tarefa não foi aplicada. Cabe ressaltar que, segundo Duval (2009), a 
passagem de um sistema de representação semiótico a outro não tem nada de espontâneo e muitos alunos podem não reconhecer o mesmo objeto em sistemas diferentes.

A análise qualitativa da sequência didática sugere que a apresentação, com os tipos de representação pouco utilizados de forma geral na escola hoje, favorece diversos fatores relativos ao conceito de soma de arcos. Concluímos que uma representação que evidencie a soma de arcos, assim como a conversão de registros, pode favorecer um melhor entendimento dos objetos com os quais se está operando e explicitando propriedades inerentes aos mesmos.

\section{Referências}

AURÉLIO, B. H. F. Minidicionário: século XXI, Rio de Janeiro: Nova Fronteira, 2000.

BERLINGOFF, W. P.; GOUVÊA, F. Q. A matemática através dos tempos: um guia fácil e prático para professores e entusiastas. Trad. Elza Gomide, Helena Castro. São Paulo: Edgard Blücher, 2008.

DUVAL, R. Semiósis e pensamento humano: registro semiótico e aprendizagens intelectuais (Sémiosis et Pensée Humaine: Registres Sémiotiques et apprentissages Intellectuels). Tradução: Lênio Fernandes Levy e Marisa Rosâni Abreu da Silveira - São Paulo: Editora Livraria da Física, 2009.

DUVAL, R. Ver e ensinar a Matemática de outra forma: entrar no modo matemático de pensar os registros de representações semióticas, - São Paulo: PROEM, 2011.

FRIZZARINI, S.T. Operações com arcos. In CARGNIN, C. et. al. Trigonometria no ensino médio: o ensino com apoio computacional. Curitiba: Appris, 2018.

JANOS, M. Matemática para pais (e) interessados. Volume 2: geometrias. São Paulo: Livraria da Física, 2011. 